\title{
Output Strictly Passive Control of Uncertain Singular Neutral Systems
}

\author{
Jichun Wang, ${ }^{1,2}$ Qingling Zhang, ${ }^{1}$ and Dong Xiao ${ }^{3}$ \\ ${ }^{1}$ Institute of Systems Science, Northeastern University, Shenyang, Liaoning 110004, China \\ ${ }^{2}$ College of Science, Liaoning University of Technology, Jinzhou, Liaoning 121001, China \\ ${ }^{3}$ Information Science and Engineering School, Northeastern University, Shenyang 110004, China
}

Correspondence should be addressed to Jichun Wang; wangjichunxd@aliyun.com

Received 18 July 2014; Accepted 23 September 2014

Academic Editor: Xi-Ming Sun

Copyright (C) 2015 Jichun Wang et al. This is an open access article distributed under the Creative Commons Attribution License, which permits unrestricted use, distribution, and reproduction in any medium, provided the original work is properly cited.

\begin{abstract}
This paper concerns the problem of output strictly passive control for uncertain singular neutral systems. It introduces a new effective criterion to study the passivity of singular neutral systems. Compared with the previous approach, this criterion has no equality constraints. And the state feedback controller is designed so that the uncertain singular neutral systems are output strictly passive. In terms of a linear matrix inequality (LMI) and Lyapunov function, the strictly passive criterion is formulated. And the desired passive controller is given. Finally, an illustrative example is given to demonstrate the effectiveness of the proposed approach.
\end{abstract}

\section{Introduction}

In many physical, industrial, and circuit systems, time delays can impose difficulties and restrictions on the design of a controller. So in these control problems, taking the delays into account is particularly important for performance evaluation and control system's design. Since 1960s, the delay control problem has attracted much attention due to its both practical and theoretical importance. Various approaches have been developed and a great number of results for continuous systems as well as discrete systems have been reported in the literature; see, for instance, [1-4]. These years some researchers found that many practical systems can be modeled by delay differential equations of neutral type [5]. This kind of system contains delays both in its state and in its derivatives of state. Such systems are often encountered in networks of interconnected systems, lossless transmission lines, partial element equivalent circuits in electrical engineering, controlled constrained manipulators in mechanical engineering [6], and certain implementation schemes of predictive controllers [7]. Very recently, interest has been focused on the study of the theory of normal neutral delay systems and some issues [8]. The problems of stability analysis and control of neutral systems have been investigated and many results on these topics have been obtained [9-11]. Mahmoud [12], Xu et al. [13], and Xia et al. [14], for example, studied $H_{\infty}$ control problem for neutral system. And Li [15], for example, studied robust control for this kind of systems. Since the 70s, the control problem of passivity [16] theory has attracted much attention due to its both practical and theoretical importance. Therefore, about passive control for neutral system, there are very few literatures. Cai-na and Baotong [17] and $\mathrm{Hu}$ et al. $[18,19]$ researched the passive control of uncertain linear neutral systems. Yang et al. [20] studied fuzzy neutral systems. However, to the best of the authors' knowledge, there are very few literatures which study singular neutral systems.

Singular systems [21] have been extensively studied in the past years due to the fact that singular systems better described physical systems than state-space ones. Therefore, the study of neutral singular system is of theoretical and practical importance. However, very little attention has been drawn to the problem of the general form of neutral type system, that is, neutral type singular systems. It is more complex and few researchers studied this sort of system. 
We know that most researchers only study the stability aspect for neutral singular systems. References [22, 23] study the stability and state feedback stabilization problems of general neutral type descriptor system with mixed delays. Reference [24] concerns the problem of the delaydependent robust stability for neutral singular systems with time-varying delays and nonlinear perturbations $[25,26]$. Reference [27] deals with the problem of robust stability of uncertain neutral singular system with neutral and discrete delays. So far, we have not found the problem of output strictly passive control about singular neutral systems.

In this paper, we are concerned with the problem of output strictly passive control for uncertain neutral singular systems. The parameter uncertainties are assumed to be timevarying and unknown but norm-bounded appearing in state matrices. Owing to the singularity of the derivative matrix $E$, most of the previous conclusions have a nonstrict inequality $E^{T} P=P^{T} E \geq 0$, which contain equality constraints. This constraint may result in numerical problems when checking such nonstrict LMI conditions since equality constraints are fragile and usually not satisfied perfectly. Therefore, strict LMI conditions are more desirable than nonstrict ones from the numerical point of view. This paper introduces a new criterion with strictly LMI which removes the equality constraints and overcomes the shortage before. Then the output strictly passive controller is designed such that the resulting closed-loop system satisfied the prescribed passive performance level. Finally, we give illustrative examples to demonstrate our proposed criteria.

Notation. Throughout this note, the following notations and conventions will be in force. The symbols $R, R_{+}, C$, and $C_{+}$denote the sets of real numbers, complex numbers, and complex numbers with positive real part, respectively. The notation $R^{n \times m}$ denotes the set of $n \times m$ matrices with real elements and $R^{n \times m}(s)$ denotes the set of $n \times m$ matrices of rational functions. Let $P$ be a square matrix. The matrix $P$ is said to be symmetric if $P=P^{T}$. For symmetric matrices $X$ and $Y$, the notation $X \geq Y$ (resp., $X>Y$ ) means that the matrix $X-Y$ is positive semidefinite (resp., positive definite). $I$ is the identity matrix with appropriate dimension. The superscripts " $T$ " and “*” represent the transpose and the complex conjugate transpose. $\|x\|$ is the Euclidean norm of the vector $x$. Matrices, if not explicitly stated, are assumed to have compatible dimensions.

\section{System Description and Preliminaries}

In this paper, we consider the following uncertain singular neutral delay system:

$$
\begin{gathered}
E \dot{x}(t)=(A+\Delta A) x(t)+\left(A_{1}+\Delta A_{1}\right) x(t-\tau) \\
+G \dot{x}(t-\tau)+B \omega(t)+B_{1} u(t), \\
y(t)=C x(t)+D \omega(t), \\
x(t)=\phi(t), \quad t \in\left[t_{0}-\tau, t_{0}\right],
\end{gathered}
$$

where $x(t) \in R^{n}$ is the state variable vector; $u(t) \in R^{m}$ is the control input vector; $y(t) \in R^{q}$ is the output vector; $\omega(t) \in L_{2}[0, \infty)$ is the disturbance input vector; $\phi(t)$ are continuous functions defined on $(-\infty, 0] . E, A, A_{1}, G, B, B_{1}$, $C, D$ are given constant matrices with appropriate dimensions and $\tau>0$ is constant time-delay. $\Delta E, \Delta A, \Delta A_{1}$ are unknown real norm-bounded matrix functions which represent timevarying parameter uncertainties. In this paper, the uncertainties are assumed to be of the form

$$
\left[\begin{array}{cc}
\Delta A & \Delta A_{1}
\end{array}\right]=H F(t)\left[\begin{array}{ll}
N_{a} & N_{a 1}
\end{array}\right]
$$

where $H, N_{a}, N_{a 1}$ are known real constant matrices with appropriate dimensions; for all $t, F(t)$ is an unknown real matrix satisfying

$$
F(t) F^{T}(t) \leq I
$$

where $I$ is unit matrix with appropriate dimensions.

Remark 1. When $E=I$, the system ((1a), (1b), and (1c)) reduces to the traditional uncertain neutral system with time delays.

The nominal unforced singular neutral system of the system ((1a), (1b), and (1c)) can be written as

$$
\begin{gathered}
E \dot{x}(t)=A x(t)+A_{1} x(t-\tau)+G \dot{x}(t-\tau)+B \omega(t), \\
y(t)=C x(t)+D \omega(t), \\
x(t)=\phi(t), \quad t \in\left[t_{0}-\tau, t_{0}\right] .
\end{gathered}
$$

In what follows, we introduce some of the data that will be used later.

Definition 2 (see [28]). Suppose $\mathfrak{\Im}: C \rightarrow R^{n}$ is linear and continuous and let $C_{\mathfrak{I}}=\{\Phi \in C: \mathfrak{I} \Phi=0\}$. The operator $\mathfrak{I}$ is said to be stable if the zero solution of the homogeneous difference equation

$$
\Im y_{t}=0, \quad t \geq 0, \quad y_{0}=\Psi \in C_{\Im},
$$

is uniformly asymptotically stable.

Lemma 3 (see [28]). The following statements are equivalent.

(1) $\mathfrak{I}$ is stable.

(2) There are constants $a>0$ and $b>0$ such that, for any $h \in C\left([0, \infty), R^{n}\right)$, any solution $y$ of the nonhomogeneous equation

$$
\Im y_{t}=h(t), \quad t \geq 0,
$$

satisfies

$$
\left|y_{t}\right| \leq b e^{-a t}\left|y_{0}\right|+b \sup _{0 \leq u \leq t}|h(u)|, \quad t \geq 0 .
$$

In order to simplify the treatment of the problem, here the operator $\mathfrak{I}: C\left([-\tau, 0], R^{n}\right) \rightarrow R^{n}$ is defined to be

$$
\mathfrak{J}\left(x_{t}\right)=E x(t)-G x(t-\tau) .
$$


Remark 4. It is noted that the regularity of the neutral singular system ((4a), (4b), and (4c)) and the stability of operator $\mathfrak{I}$ can ensure the existence and uniqueness of the solution, which is shown in the following lemma.

Lemma 5. If the pair $(E, A)$ is regular and $\operatorname{det}\left(E-e^{-s \tau} G\right) \neq 0$, then the solution to the neutral singular system ((4a), (4b), and $(4 c))$ exists and is unique on $(0, \infty)$.

Proof. The regularity of the pair $(E, A)$ is discussed in the book [29]. Take the Laplace transform for the equation

$$
E x(t)-G x(t-\tau)=0 .
$$

Then obtain the desired result immediately.

In this paper, we emphasize on the case that the solution to the neutral singular system exists and is unique. And we always assume $\left(D+D^{T}\right)>0$.

Lemma 6 (see [30]). Let $A, L, E$, and $F$ be real matrices of appropriate dimensions, with $F$ satisfying $F^{T} F \leq I$. Then one has the following.

(1) For any scalar $\varepsilon>0$,

$$
L F E+E^{T} F^{T} L^{T} \leq \varepsilon^{-1} L L^{T}+\varepsilon E^{T} E .
$$

(2) For any matrix $P>0$ and scalar $\varepsilon>0$ such that $\varepsilon I-$ $E F E^{T}>0$,

$$
\begin{aligned}
(A & +L F E)^{T} P(A+L F E) \\
& \leq A^{T} P A+A^{T} P E\left(\varepsilon I-E^{T} P E\right)^{-1} E^{T} P A+\varepsilon L^{T} L .
\end{aligned}
$$

Our problem is to establish the output and input strictly passive controller for the system ((1a), (1b), and (1c)) to determine the conditions. First, we introduce the following definitions of passivity.

Definition 7 (see [31]). The dynamical system ((4a), (4b), and $(4 \mathrm{c}))$ is called passive if

$$
\int_{0}^{\infty} \omega^{T}(t) y(t) d t>\beta, \quad \forall \omega \in L_{2}[0, \infty)
$$

where $\beta$ is some constant which depends on the initial condition of the system. In addition, the system is said to be output strictly passive (OSP) if

$$
\int_{0}^{\infty}\left[\omega^{T}(t) y(t)-\delta_{0}\|y(t)\|^{2}\right] d t>\beta, \quad \forall \omega \in L_{2}[0, \infty) .
$$

And the system is said to be input strictly passive (ISP) if

$$
\int_{0}^{\infty}\left[\omega^{T}(t) y(t)-\delta_{0}\|u(t)\|^{2}\right] d t>\beta, \quad \forall \omega \in L_{2}[0, \infty) .
$$

Here $\delta_{0}>0$ is a scalar.
For the system ((1a), (1b), and (1c)), we are interested in designing a state feedback controller of the form

$$
u(t)=K_{a} x(t)+K_{a 1} x(t-\tau),
$$

where the state feedback gains $K_{a}$ and $K_{a 1}$ are appropriate constant matrices. Since gain perturbations may arise when implementing the controller (15) into the system ((1a), (1b), and (1c)), the actual controller will be of the following form:

$$
u(t)=\left(K_{a}+\Delta K_{a}(t)\right) x(t)+\left(K_{a 1}+\Delta K_{a 1}(t)\right) x(t-\tau),
$$

where $\Delta K_{a}(t)$ and $\Delta K_{a 1}(t)$ are the controller gain perturbation. There are two types of perturbations considered in this paper.

(1) $\Delta K_{a}(t)$ and $\Delta K_{a 1}(t)$ are with the norm-bounded additive form; that is, the perturbations are independent of matrices $K_{a}$ and $K_{a 1}$, respectively,

$$
\left[\begin{array}{ll}
\Delta K_{a}(t) & \Delta K_{a 1}(t)
\end{array}\right]=M_{a} F_{a}(t)\left[\begin{array}{ll}
L_{a} & L_{a 1}
\end{array}\right],
$$

where $M_{a}, L_{a}$, and $L_{a 1}$ are known matrices and $F_{a}(t)$ is an unknown matrix satisfying

$$
F_{a}^{T}(t) F_{a}(t) \leq I .
$$

(2) $\Delta K_{a}(t)$ and $\Delta K_{a 1}(t)$ are with the norm-bounded multiplicative form; that is, the perturbations are dependent on matrices $K_{a}$ and $K_{a 1}$, respectively,

$$
\left[\begin{array}{ll}
\Delta K_{a}(t) & \Delta K_{a 1}(t)
\end{array}\right]=H_{m} F_{m}(t)\left[\begin{array}{lll}
L_{m} K_{a} & L_{m} K_{a 1}
\end{array}\right],
$$

where $H_{m}$ and $L_{m}$ are known matrices and $F_{m}(t)$ is an unknown matrix satisfying

$$
F_{m}^{T}(t) F_{m}(t) \leq I
$$

When we apply the controller (16) to system ((1a), (1b), and (1c)), the resulting closed-loop system is written as

$$
E \dot{x}(t)=A_{c} x(t)+A_{1 c} x(t-\tau)+G \dot{x}(t-\tau)+B \omega(t),
$$

where

$$
\begin{gathered}
A_{c}=A+\Delta A+B_{1}\left(K_{a}+\Delta K_{a}(t)\right), \\
A_{1 c}=A_{1}+\Delta A+B_{1}\left(K_{a 1}+\Delta K_{a 1}(t)\right) .
\end{gathered}
$$

In this section, a controller (16) with (17) and (19) will be designed and satisfy the following conditions:

(1) with $\omega(t)=0$, the closed-loop system (21) is asymptotically stable;

(2) with $\omega(t) \neq 0$, the following inequality

$$
\int_{0}^{\infty}\left[\omega^{T}(t) y(t)-\delta_{0}\|y(t)\|^{2}\right] d t>\beta, \quad \forall \omega \in L_{2}[0, \infty),
$$

holds for all trajectories with zero initial condition and some $\delta_{0}$. The system satisfying this condition is said to be output strictly passive. The obtained controller (16) is said to be output strictly passive controller of system ((1a), (1b), and (1c)). 


\section{Main Results}

Firstly, we analysis the output strictly passivity for the uncontrolled nominal singular neutral system ((4a), (4b), and (4c)).

Theorem 8. Consider a state-delay neutral singular system ((4a), (4b), and (4c)), given the scalar $\delta_{0}>0$, if there exist positive definite matrices $P$ and $Q$, matrices $N_{1}, N_{2}$ with appropriate dimensions, and scalars $\varepsilon_{1}>0, \varepsilon_{2}>0$ satisfying the following linear matrix inequality (LMI):

$$
\left[\begin{array}{ccccccc}
\Pi_{1} & \Pi_{2} & E^{T} P B-C^{T}+2 \delta_{0} C^{T} D & N_{1} & 0 & \varepsilon_{1} I & \varepsilon_{2} I \\
* & \Pi_{3} & -G^{T} P B & 0 & N_{2} & -\varepsilon_{1} I & -\varepsilon_{2} I \\
* & * & -D-D^{T}+2 \delta_{0} D^{T} D & 0 & 0 & 0 & 0 \\
* & * & * & -\varepsilon_{1} I & 0 & 0 & 0 \\
* & * & * & * & -\varepsilon_{2} I & 0 & 0 \\
* & * & * & * & * & -\varepsilon_{1} I & 0 \\
* & * & * & * & * & * & -\varepsilon_{2} I
\end{array}\right]
$$

$<0$,

where

$$
\begin{aligned}
& \Pi_{1}=A^{T} P E+E^{T} P A+N_{1}+N_{1}^{T}+2 \delta_{0} C^{T} C+Q, \\
& \Pi_{2}=-A^{T} P G+E^{T} P A_{1}-N_{1}+N_{2}^{T}, \\
& \Pi_{3}=-A_{1}^{T} P G-G^{T} P A_{1}-N_{2}-N_{2}^{T}-Q .
\end{aligned}
$$

Then the system ((4a), (4b), and (4c)) is output strictly passive for the time-delay $\tau$.

Proof. Define a Lyapunov functional $V(x(t))$ as follows:

$$
\begin{aligned}
& V(x(t))=V_{1}\left(x_{t}\right)+V_{2}\left(x_{t}\right), \\
& V_{1}\left(x_{t}\right)=\mathfrak{J}^{T}\left(x_{t}\right) P \mathfrak{J}\left(x_{t}\right), \\
& V_{2}\left(x_{t}\right)=\int_{t-\tau}^{t} x^{T}(\theta) Q x(\theta) d \theta .
\end{aligned}
$$

For any matrices $N_{1}$ and $N_{2}$ of appropriate dimension, we have

$$
\begin{aligned}
& 2\left[x^{T}(t) N_{1}+x^{T}(t-\tau) N_{2}\right] \\
& \quad \times\left[x(t)-x(t-\tau)-\int_{t-\tau}^{t} \dot{x}(\theta) d \theta\right]=0 .
\end{aligned}
$$

Calculating the derivative of the Lyapunov function $V(x(t))$ along the system ((4a), (4b), and (4c)) and adding (27), it gives

$$
\left.\begin{array}{cc}
\Pi_{2}-\left(\varepsilon_{1}^{-1}+\varepsilon_{2}^{-1}\right) I & E^{T} P B \\
\Pi_{1}+\varepsilon_{2} N_{2} N_{2}^{T}+\left(\varepsilon_{1}^{-1}+\varepsilon_{2}^{-1}\right) I & -G^{T} P B \\
* & 0
\end{array}\right]\left[\begin{array}{c}
x(t) \\
x(t-\tau) \\
\omega(t)
\end{array}\right]
$$

when $B=0$; if (24) holds, it is obvious that the system ((4a), (4b), and (4c)) is asymptotically stable.
Through these we can obtain that

$$
\begin{aligned}
& \dot{V}(x(t))-2\left[\omega^{T}(t) y(t)-\delta_{0}\|y(t)\|^{2}\right] \\
& \quad \leq\left[\begin{array}{c}
x(t) \\
x(t-\tau) \\
\omega(t)
\end{array}\right]\left[\begin{array}{ccc}
\Pi_{1}+\varepsilon_{1} N_{1} N_{1}^{T}+\left(\varepsilon_{1}^{-1}+\varepsilon_{2}^{-1}\right) I & \Pi_{2}-\left(\varepsilon_{1}^{-1}+\varepsilon_{2}^{-1}\right) I & E^{T} P B-C^{T}+2 \delta_{0} C^{T} D \\
* & \Pi_{1}+\varepsilon_{2} N_{2} N_{2}^{T}+\left(\varepsilon_{1}^{-1}+\varepsilon_{2}^{-1}\right) I & -G^{T} P B \\
* & * & -D-D^{T}+2 \delta_{0} D^{T} D
\end{array}\right]\left[\begin{array}{c}
x(t) \\
x(t-\tau) \\
\omega(t)
\end{array}\right] .
\end{aligned}
$$

Hence, if (24) holds, then

$$
\dot{V}(x(t))<2\left[\omega^{T}(t) y(t)-\delta_{0}\|y(t)\|^{2}\right] .
$$

Integrating (30) from $t_{0}$ to $t_{1}$, we have

$$
\begin{array}{r}
\int_{t_{0}}^{t_{1}}\left[\omega^{T}(t) y(t)-\delta_{0}\|y(t)\|^{2}\right] d t \\
>\frac{1}{2}\left[V\left(x\left(t_{1}\right)\right)-V\left(x\left(t_{0}\right)\right)\right] .
\end{array}
$$

Since $V(x(t))>0$ for $x \neq 0$ and $V(x(t))=0$ for $x=0$, it follows that as $t_{0}=0$ and $t_{1} \rightarrow \infty$ the system ((4a), (4b), and $(4 c))$ is output strictly passive.

Remark 9. In recent years, LMI has become the main method to solve the control problem of singular systems. Because of the singularity of $E$, almost all results have a nonstrict inequality $E^{T} P=P^{T} E \geq 0$, which contain equality constraints. These constraints may result in numerical problems 
when checking such nonstrict LMI conditions since equality constraints are fragile and usually not satisfied perfectly. Therefore, strict LMI conditions are more desirable than nonstrict ones from the numerical point of view. In this paper, the conclusions obtained are strict LMI, which remove the equality constraints and overcome the shortcoming before.

Corollary 10. Consider a state-delay neutral singular system ((4a), (4b), and (4c)), given the scalar $\delta_{0}>0$, if there exist positive definite matrices $P$ and $Q$, matrices $N_{1}, N_{2}$ with appropriate dimensions, and scalars $\varepsilon_{1}>0, \varepsilon_{2}>0$ satisfying the following linear matrix inequality (LMI):

$$
\left[\begin{array}{ccccccc}
\widehat{\Pi}_{1} & \widehat{\Pi}_{2} & E P C^{T}-B+2 \delta_{0} B D^{T} & N_{1} & 0 & \varepsilon_{1} I & \varepsilon_{2} I \\
* & \widehat{\Pi}_{3} & -G P C^{T} & 0 & N_{2} & -\varepsilon_{1} I & -\varepsilon_{2} I \\
* & * & -D-D^{T}+2 \delta_{0} D D^{T} & 0 & 0 & 0 & 0 \\
* & * & * & -\varepsilon_{1} I & 0 & 0 & 0 \\
* & * & * & * & -\varepsilon_{2} I & 0 & 0 \\
* & * & * & * & * & -\varepsilon_{1} I & 0 \\
* & * & * & * & * & * & -\varepsilon_{2} I
\end{array}\right]
$$

$<0$ where

$$
\begin{aligned}
& \widehat{\Pi}_{1}=A P E^{T}+E P A^{T}+N_{1}+N_{1}^{T}+2 \delta_{0} B B^{T}+Q, \\
& \widehat{\Pi}_{2}=-A P G^{T}+E P A_{1}^{T}-N_{1}+N_{2}^{T}, \\
& \widehat{\Pi}_{3}=-A_{1} P G^{T}-G P A_{1}^{T}-N_{2}-N_{2}^{T}-Q .
\end{aligned}
$$

Then the system ((4a), (4b), and (4c)) is output strictly passive for the time-delay $\tau$.

Proof. The system ((4a), (4b), and (4c)) and its dual system have the same passivity. Therefore, according to $\mathrm{Xu}$ [32] obtain the conclusion (32) and (33).

Remark 11. The transfer functions of system ((4a), (4b), and (4c)) and its dual system are transposed each other, so they have the same properties.

Theorem 12. Consider a state-delay uncertain neutral singular system ((4a), (4b), and (4c)), given the scalar $\delta_{0}>0$, if there exist positive definite matrices $P$ and $Q$, matrices $N_{1}, N_{2}$ with appropriate dimensions, and scalars $\varepsilon_{i}>0, i=1, \ldots, 6$, satisfying the following linear matrix inequality (LMI):

$$
\left[\begin{array}{ccccccccccccccc}
\Sigma_{1} & \Sigma_{2} & \Sigma_{3} & N_{1} & 0 & \varepsilon_{1} I & \varepsilon_{2} I & \Sigma_{7} & \Sigma_{8} & 0 & 0 & \varepsilon_{3} H & \varepsilon_{4} H & 0 & 0 \\
* & \Sigma_{4} & \Sigma_{5} & 0 & N_{2} & -\varepsilon_{1} I & -\varepsilon_{2} I & 0 & 0 & \Sigma_{9} & \Sigma_{10} & 0 & 0 & \varepsilon_{5} H & \varepsilon_{6} H \\
* & * & \Sigma_{6} & 0 & 0 & 0 & 0 & 0 & 0 & 0 & 0 & 0 & 0 & 0 & 0 \\
* & * & * & -\varepsilon_{1} I & 0 & 0 & 0 & 0 & 0 & 0 & 0 & 0 & 0 & 0 & 0 \\
* & * & * & * & -\varepsilon_{2} I & 0 & 0 & 0 & 0 & 0 & 0 & 0 & 0 & 0 & 0 \\
* & * & * & * & * & -\varepsilon_{1} I & 0 & 0 & 0 & 0 & 0 & 0 & 0 & 0 & 0 \\
* & * & * & * & * & * & -\varepsilon_{2} I & 0 & 0 & 0 & 0 & 0 & 0 & 0 & 0 \\
* & * & * & * & * & * & * & -\varepsilon_{3} I & 0 & 0 & 0 & 0 & 0 & 0 & 0 \\
* & * & * & * & * & * & * & * & -\varepsilon_{5} I & 0 & 0 & 0 & 0 & 0 & 0 \\
* & * & * & * & * & * & * & * & * & -\varepsilon_{4} I & 0 & 0 & 0 & 0 & 0 \\
* & * & * & * & * & * & * & * & * & * & -\varepsilon_{6} I & 0 & 0 & 0 & 0 \\
* & * & * & * & * & * & * & * & * & * & * & -\varepsilon_{3} I & 0 & 0 & 0 \\
* & * & * & * & * & * & * & * & * & * & * & * & -\varepsilon_{4} I & 0 & 0 \\
* & * & * & * & * & * & * & * & * & * & * & * & * & -\varepsilon_{5} I & 0 \\
* & * & * & * & * & * & * & * & * & * & * & * & * & * & -\varepsilon_{6} I
\end{array}\right]<0,
$$

where

$$
\begin{aligned}
& \Sigma_{1}=A P E^{T}+E P A^{T}+N_{1}+N_{1}^{T}+2 \delta_{0} B B^{T}+Q, \\
& \Sigma_{2}=-A P G^{T}+E P A_{1}^{T}-N_{1}+N_{2}^{T}, \\
& \Sigma_{3}=E P C^{T}-B+2 \delta_{0} B D^{T}, \\
& \Sigma_{4}=-A_{1} P G^{T}-G P A_{1}^{T}-N_{2}-N_{2}^{T}-Q, \\
& \Sigma_{5}=-G P C^{T},
\end{aligned}
$$

$$
\begin{aligned}
& \Sigma_{6}=-D-D^{T}+2 \delta_{0} D D^{T}, \\
& \Sigma_{7}=E P N_{a}^{T}, \\
& \Sigma_{8}=E P N_{a 1}^{T}, \\
& \Sigma_{9}=G P N_{a}^{T}, \\
& \Sigma_{10}=G P N_{a 1}^{T} .
\end{aligned}
$$

Then the system ((1a), (1b), and (1c)) is output strictly passive for the time-delay $\tau$. 
Proof. Let $\varepsilon=\varepsilon_{1}^{-1}+\varepsilon_{2}^{-1}$. Denote

$$
\Xi=\left[\begin{array}{ccccc}
A P E^{T}+E P A^{T}+N_{1}+N_{1}^{T}+2 \delta_{0} B B^{T}+\varepsilon I+Q & -A P G^{T}+E P A_{1}^{T}-N_{1}+N_{2}^{T}-\varepsilon I & E P C^{T}-B+2 \delta_{0} B D^{T} & N_{1} & 0 \\
* & -A_{1} P G^{T}-G P A_{1}^{T}-N_{2}-N_{2}^{T}-Q+\varepsilon I & -G P C^{T} & 0 & N_{2} \\
* & * & -D-D^{T}+2 \delta_{0} D D^{T} & 0 & 0 \\
* & * & * & -\varepsilon_{1} I & 0 \\
* & * & * & * & -\varepsilon_{2} I
\end{array}\right] .
$$

Applying the Schur complement formula to (34), we can obtain

$$
\begin{aligned}
& \Xi+\left(\varepsilon_{3}^{-1}+\varepsilon_{4}^{-1}\right)\left[\begin{array}{c}
H \\
0 \\
0 \\
0 \\
0
\end{array}\right]\left[\begin{array}{c}
H \\
0 \\
0 \\
0 \\
0
\end{array}\right]^{T}+\left(\varepsilon_{5}^{-1}+\varepsilon_{6}^{-1}\right)\left[\begin{array}{c}
0 \\
H \\
0 \\
0 \\
0
\end{array}\right]\left[\begin{array}{c}
0 \\
H \\
0 \\
0 \\
0
\end{array}\right]^{T} \\
& +\varepsilon_{3}\left[\begin{array}{c}
E P N_{a}^{T} \\
0 \\
0 \\
0 \\
0
\end{array}\right]\left[\begin{array}{c}
E P N_{a}^{T} \\
0 \\
0 \\
0 \\
0
\end{array}\right]^{T} \\
& +\varepsilon_{5}\left[\begin{array}{c}
E P N_{a 1}^{T} \\
0 \\
0 \\
0 \\
0
\end{array}\right]\left[\begin{array}{c}
E P N_{a 1}^{T} \\
0 \\
0 \\
0 \\
0
\end{array}\right]^{T}+\varepsilon_{4}\left[\begin{array}{c}
0 \\
G P N_{a}^{T} \\
0 \\
0 \\
0
\end{array}\right]\left[\begin{array}{c}
0 \\
G P N_{a}^{T} \\
0 \\
0 \\
0
\end{array}\right]^{T} \\
& +\varepsilon_{6}\left[\begin{array}{c}
0 \\
G P N_{a 1}^{T} \\
0 \\
0 \\
0
\end{array}\right]\left[\begin{array}{c}
0 \\
G P N_{a 1}^{T} \\
0 \\
0 \\
0
\end{array}\right]^{T}<0
\end{aligned}
$$

which, by Lemma 6, holds if and only if

$$
\Xi+\Omega_{1}+\Omega_{1}^{T}+\Omega_{2}+\Omega_{2}^{T}+\Omega_{3}+\Omega_{3}^{T}+\Omega_{4}+\Omega_{4}^{T}<0,
$$

where

$$
\Omega_{1}=\left[\begin{array}{c}
H \\
0 \\
0 \\
0 \\
0
\end{array}\right] F(t)\left[N_{a} P E^{T} \quad 0 \quad 0 \quad 00\right],
$$

$$
\begin{aligned}
& \Omega_{2}=\left[\begin{array}{c}
-H \\
0 \\
0 \\
0 \\
0
\end{array}\right] F(t)\left[\begin{array}{lllll}
0 & N_{a} P G^{T} & 0 & 0 & 0
\end{array}\right], \\
& \Omega_{3}=\left[\begin{array}{c}
0 \\
H \\
0 \\
0 \\
0
\end{array}\right] F(t)\left[N_{a 1} P E^{T} \quad 0 \quad 0 \quad 00\right] \text {, } \\
& \Omega_{4}=\left[\begin{array}{c}
0 \\
-H \\
0 \\
0 \\
0
\end{array}\right] F(t)\left[\begin{array}{lllll}
0 & N_{a 1} P G^{T} & 0 & 0 & 0
\end{array}\right] .
\end{aligned}
$$

In terms of Corollary 10 we will get the desired conclusion.

The following results present the solvability conditions for the state feedback output strictly passive control problem for the uncertain neutral singular systems ((1a), (1b), and (1c)) with the controller perturbation in (17) and (18), or (19) and (20), respectively.

First, we discuss the output strictly passive controller (16) with the norm-bounded additive form perturbation in (17) and (18).

Theorem 13. Consider a state-delay uncertain neutral singular system ((1a), (1b), and (1c)); there exists a controller (16) with additive gain perturbation in (17) and (18) such that the resulting closed-loop system (21) is output strictly passive for the time-delay $\tau$ with dissipation $\delta_{0}>0$ for all nonzero $\omega(t) \in L_{2}[0, \infty)$, if there exist positive definite matrices $P$ and $Q$, matrices $N_{1}, N_{2}, T_{1}, T_{2}$ with appropriate dimensions, and 
scalars $\varepsilon_{i}>0, i=1, \ldots, 10$, satisfying the following linear matrix inequality (LMI):

$$
\begin{aligned}
& {\left[\begin{array}{cccccccccccc}
\Upsilon_{1} & \Upsilon_{2} & \Sigma_{3} & N_{1} & 0 & \varepsilon_{1} I & \varepsilon_{2} I & \Sigma_{7} & \Sigma_{8} & 0 & 0 & \varepsilon_{3} H \\
* & \Upsilon_{3} & \Sigma_{5} & 0 & N_{2} & -\varepsilon_{1} I & -\varepsilon_{2} I & 0 & 0 & \Sigma_{9} & \Sigma_{10} & 0 \\
* & * & \Sigma_{6} & 0 & 0 & 0 & 0 & 0 & 0 & 0 & 0 & 0 \\
* & * & * & -\varepsilon_{1} I & 0 & 0 & 0 & 0 & 0 & 0 & 0 & 0 \\
* & * & * & * & -\varepsilon_{2} I & 0 & 0 & 0 & 0 & 0 & 0 & 0 \\
* & * & * & * & * & -\varepsilon_{1} I & 0 & 0 & 0 & 0 & 0 & 0 \\
* & * & * & * & * & * & -\varepsilon_{2} I & 0 & 0 & 0 & 0 & 0 \\
* & * & * & * & * & * & * & -\varepsilon_{3} I & 0 & 0 & 0 & 0 \\
* & * & * & * & * & * & * & * & -\varepsilon_{5} I & 0 & 0 & 0 \\
* & * & * & * & * & * & * & * & * & -\varepsilon_{4} I & 0 & 0 \\
* & * & * & * & * & * & * & * & * & * & -\varepsilon_{6} I & 0 \\
* & * & * & * & * & * & * & * & * & * & * & -\varepsilon_{3} I \\
* & * & * & * & * & * & * & * & * & * & * & * \\
* & * & * & * & * & * & * & * & * & * & * & * \\
* & * & * & * & * & * & * & * & * & * & * & * \\
* & * & * & * & * & * & * & * & * & * & * & * \\
* & * & * & * & * & * & * & * & * & * & * & * \\
* & * & * & * & * & * & * & * & * & * & * & * \\
* & * & * & * & * & * & * & * & * & * & * & * \\
* & * & * & * & * & * & * & * & * & * & * & * \\
* & * & * & * & * & * & * & * & * & * & * & * \\
* & * & * & * & * & * & * & * & * & * & * & * \\
* & * & * & * & * & * & * & * & * & * & * & *
\end{array}\right.} \\
& \left.\begin{array}{ccccccccccc}
\varepsilon_{4} H & 0 & 0 & Z_{4} & 0 & 0 & 0 & 0 & \varepsilon_{7} Z_{1} & \varepsilon_{9} Z_{1} & \varepsilon_{10} Z_{1} \\
0 & \varepsilon_{5} H & \varepsilon_{6} H & 0 & Z_{5} & Z_{2} & Z_{3} & \varepsilon_{8} Z_{1} & 0 & 0 & 0 \\
0 & 0 & 0 & 0 & 0 & 0 & 0 & 0 & 0 & 0 & 0 \\
0 & 0 & 0 & 0 & 0 & 0 & 0 & 0 & 0 & 0 & 0 \\
0 & 0 & 0 & 0 & 0 & 0 & 0 & 0 & 0 & 0 & 0 \\
0 & 0 & 0 & 0 & 0 & 0 & 0 & 0 & 0 & 0 & 0 \\
0 & 0 & 0 & 0 & 0 & 0 & 0 & 0 & 0 & 0 & 0 \\
0 & 0 & 0 & 0 & 0 & 0 & 0 & 0 & 0 & 0 & 0 \\
0 & 0 & 0 & 0 & 0 & 0 & 0 & 0 & 0 & 0 & 0 \\
0 & 0 & 0 & 0 & 0 & 0 & 0 & 0 & 0 & 0 & 0 \\
0 & 0 & 0 & 0 & 0 & 0 & 0 & 0 & 0 & 0 & 0 \\
0 & 0 & 0 & 0 & 0 & 0 & 0 & 0 & 0 & 0 & 0 \\
-\varepsilon_{4} I & 0 & 0 & 0 & 0 & 0 & 0 & 0 & 0 & 0 & 0 \\
* & -\varepsilon_{5} I & 0 & 0 & 0 & 0 & 0 & 0 & 0 & 0 & 0 \\
* & * & -\varepsilon_{6} I & 0 & 0 & 0 & 0 & 0 & 0 & 0 & 0 \\
* & * & * & -\varepsilon_{7} I & 0 & 0 & 0 & 0 & 0 & 0 & 0 \\
* & * & * & * & -\varepsilon_{10} I & 0 & 0 & 0 & 0 & 0 & 0 \\
* & * & * & * & * & -\varepsilon_{9} I & 0 & 0 & 0 & 0 & 0 \\
* & * & * & * & * & * & -\varepsilon_{8} I & 0 & 0 & 0 & 0 \\
* & * & * & * & * & * & * & -\varepsilon_{8} I & 0 & 0 & 0 \\
* & * & * & * & * & * & * & * & -\varepsilon_{7} I & 0 & 0 \\
* & * & * & * & * & * & * & * & * & -\varepsilon_{9} I & 0 \\
* & * & * & * & * & * & * & * & * & * & -\varepsilon_{10} I
\end{array}\right]<0,
\end{aligned}
$$


where

$$
\begin{aligned}
& \Upsilon_{1}=\Sigma_{1}+B_{1} T_{1} E^{T}+E T_{1}^{T} B_{1}^{T}, \\
& \Upsilon_{2}=\Sigma_{2}-B_{1} T_{1} G^{T}+E T_{2}^{T} B_{1}^{T}, \\
& \Upsilon_{3}=\Sigma_{4}-B_{1} T_{2} G^{T}-G T_{2}^{T} B_{1}^{T}, \\
& Z_{1}=B_{1} M_{a}, \\
& Z_{2}=G P L_{a}^{T}, \\
& Z_{3}=G P L_{a 1}^{T}, \\
& Z_{4}=E P L_{a}^{T}, \\
& Z_{5}=E P L_{a 1}^{T} .
\end{aligned}
$$

In this case, the output strictly passive controller gain can be chosen as

$$
K_{a}=T_{1} P^{-1}, \quad K_{a 1}=T_{2} P^{-1} .
$$

Proof. In terms of Theorem 12, Lemma 6, and schur complement formula, let

$$
T_{1}=K_{a} P, \quad T_{2}=K_{a 1} P .
$$

Then get the conclusion.

Next, we consider the output strictly passive controller (16) with the norm-bounded multiplicative form perturbation in (19) and (20).

Theorem 14. Consider a state-delay uncertain neutral singular system ((1a), (1b), and (1c)); there exists a controller (16) with multiplicative gain perturbation in (19) and (20) such that the resulting closed-loop system (21) is output strictly passive for the time-delay $\tau$ with dissipation $\delta_{0}>0$ for all nonzero $\omega(t) \in L_{2}[0, \infty)$, if there exist positive definite matrices $P$ and $Q$, matrices $N_{1}, N_{2}, T_{1}, T_{2}$ with appropriate dimensions, and scalars $\varepsilon_{i}>0, i=1, \ldots, 10$, satisfying the following linear matrix inequality (LMI):

$$
\left[\begin{array}{cccccccccccc}
\Upsilon_{1} & \Upsilon_{2} & \Sigma_{3} & N_{1} & 0 & \varepsilon_{1} I & \varepsilon_{2} I & \Sigma_{7} & \Sigma_{8} & 0 & 0 & \varepsilon_{3} H \\
* & \Upsilon_{3} & \Sigma_{5} & 0 & N_{2} & -\varepsilon_{1} I & -\varepsilon_{2} I & 0 & 0 & \Sigma_{9} & \Sigma_{10} & 0 \\
* & * & \Sigma_{6} & 0 & 0 & 0 & 0 & 0 & 0 & 0 & 0 & 0 \\
* & * & * & -\varepsilon_{1} I & 0 & 0 & 0 & 0 & 0 & 0 & 0 & 0 \\
* & * & * & * & -\varepsilon_{2} I & 0 & 0 & 0 & 0 & 0 & 0 & 0 \\
* & * & * & * & * & -\varepsilon_{1} I & 0 & 0 & 0 & 0 & 0 & 0 \\
* & * & * & * & * & * & -\varepsilon_{2} I & 0 & 0 & 0 & 0 & 0 \\
* & * & * & * & * & * & * & -\varepsilon_{3} I & 0 & 0 & 0 & 0 \\
* & * & * & * & * & * & * & * & -\varepsilon_{5} I & 0 & 0 & 0 \\
* & * & * & * & * & * & * & * & * & -\varepsilon_{4} I & 0 & 0 \\
* & * & * & * & * & * & * & * & * & * & -\varepsilon_{6} I & 0 \\
* & * & * & * & * & * & * & * & * & * & * & -\varepsilon_{3} I \\
* & * & * & * & * & * & * & * & * & * & * & * \\
* & * & * & * & * & * & * & * & * & * & * & * \\
* & * & * & * & * & * & * & * & * & * & * & * \\
* & * & * & * & * & * & * & * & * & * & * & * \\
* & * & * & * & * & * & * & * & * & * & * & * \\
* & * & * & * & * & * & * & * & * & * & * & * \\
* & * & * & * & * & * & * & * & * & * & * & * \\
* & * & * & * & * & * & * & * & * & * & * & * \\
* & * & * & * & * & * & * & * & * & * & * & * \\
* & * & * & * & * & * & * & * & * & * & * & * \\
* & * & * & * & * & * & * & * & * & * & * & *
\end{array}\right.
$$




$$
\left.\begin{array}{ccccccccccc}
\varepsilon_{4} H & 0 & 0 & \widehat{Z}_{4} & 0 & 0 & 0 & 0 & \varepsilon_{7} \widehat{Z}_{1} & \varepsilon_{9} \widehat{Z}_{1} & \varepsilon_{10} \widehat{Z}_{1} \\
0 & \varepsilon_{5} H & \varepsilon_{6} H & 0 & \bar{Z}_{5} & \widehat{Z}_{2} & \widehat{Z}_{3} & \varepsilon_{8} \widehat{Z}_{1} & 0 & 0 & 0 \\
0 & 0 & 0 & 0 & 0 & 0 & 0 & 0 & 0 & 0 & 0 \\
0 & 0 & 0 & 0 & 0 & 0 & 0 & 0 & 0 & 0 & 0 \\
0 & 0 & 0 & 0 & 0 & 0 & 0 & 0 & 0 & 0 & 0 \\
0 & 0 & 0 & 0 & 0 & 0 & 0 & 0 & 0 & 0 & 0 \\
0 & 0 & 0 & 0 & 0 & 0 & 0 & 0 & 0 & 0 & 0 \\
0 & 0 & 0 & 0 & 0 & 0 & 0 & 0 & 0 & 0 & 0 \\
0 & 0 & 0 & 0 & 0 & 0 & 0 & 0 & 0 & 0 & 0 \\
0 & 0 & 0 & 0 & 0 & 0 & 0 & 0 & 0 & 0 & 0 \\
0 & 0 & 0 & 0 & 0 & 0 & 0 & 0 & 0 & 0 & 0 \\
0 & 0 & 0 & 0 & 0 & 0 & 0 & 0 & 0 & 0 & 0 \\
-\varepsilon_{4} I & 0 & 0 & 0 & 0 & 0 & 0 & 0 & 0 & 0 & 0 \\
* & -\varepsilon_{5} I & 0 & 0 & 0 & 0 & 0 & 0 & 0 & 0 & 0 \\
* & * & -\varepsilon_{6} I & 0 & 0 & 0 & 0 & 0 & 0 & 0 & 0 \\
* & * & * & -\varepsilon_{7} I & 0 & 0 & 0 & 0 & 0 & 0 & 0 \\
* & * & * & * & -\varepsilon_{10} I & 0 & 0 & 0 & 0 & 0 & 0 \\
* & * & * & * & * & -\varepsilon_{9} I & 0 & 0 & 0 & 0 & 0 \\
* & * & * & * & * & * & -\varepsilon_{8} I & 0 & 0 & 0 & 0 \\
* & * & * & * & * & * & * & -\varepsilon_{8} I & 0 & 0 & 0 \\
* & * & * & * & * & * & * & * & -\varepsilon_{7} I & 0 & 0 \\
* & * & * & * & * & * & * & * & * & -\varepsilon_{9} I & 0 \\
* & * & * & * & * & * & * & * & * & * & -\varepsilon_{10} I
\end{array}\right]<,
$$

where

$$
\begin{aligned}
& \widehat{Z}_{1}=B_{1} H_{m}, \\
& \widehat{Z}_{2}=G T_{1}^{T} L_{m}^{T}, \\
& \widehat{Z}_{3}=G T_{2}^{T} L_{m}^{T}, \\
& \widehat{Z}_{4}=E T_{1}^{T} L_{m}^{T}, \\
& \widehat{Z}_{5}=E T_{2}^{T} L_{m}^{T} .
\end{aligned}
$$

Proof. The proof can be carried out by following similar line as in the proof of Theorem 13 and thus is omitted.

Remark 15. The obtained results are all independent of the time delay. When the time-delay factor is known, the delaydependent passivity [33] yields less conservative performance results, which will be studied for descriptor systems in further research.

\section{Simulation Example}

The dissolving tank is a kind of airtight container which can work under high pressure. It plays an important role in the field of civil, industrial, military, and scientific research. It is used mostly in the chemical industry among them. This paper establishes the system model based on the bipolar dissolving tank in chemical process.

Considering the bipolar dissolving tank (DT) in chemical process as shown in Figure 1, the solute in the hopper is transported to feeding throats by conveyer belt. If the feeding quantity is changed, the concentration of the solution will change in DT1. Thus the concentration of the solution in DT2 will change too, and it is also controlled by concentration of thin liquid. Generally, the thin liquid flow rate in DT1 is constant, and the thin liquid flow rate in DT2 and the feed quantity in hopper are controlled. Let the two volumes of solution for dissolving tank be $V$, and let the concentration of the solution of DT1 and DT2 be $\rho_{1}(t), \rho_{2}(t)$, respectively. Suppose the feed quantity in hopper is $u_{1}(t)$, the thin liquid flow into DT2 is $u_{2}(t)$, and the thin liquid flow into DT1 is a constant $a$. Owing to the feeding quality from the hopper to DT1 and the solution from DT1 to DT2 having a certain time, therefore the concentration of the solution of dissolving tank has lagged behind. Here we suppose the delays have all the same time $\tau$. Due to some external factors and the structure uncertainties, input disturbances are set to be $\omega(t)$. The concentration of the solution into DT1 and DT2 is $\rho_{10}(t)$ and $\rho_{20}(t)$, respectively, when the system is balanced. Let 


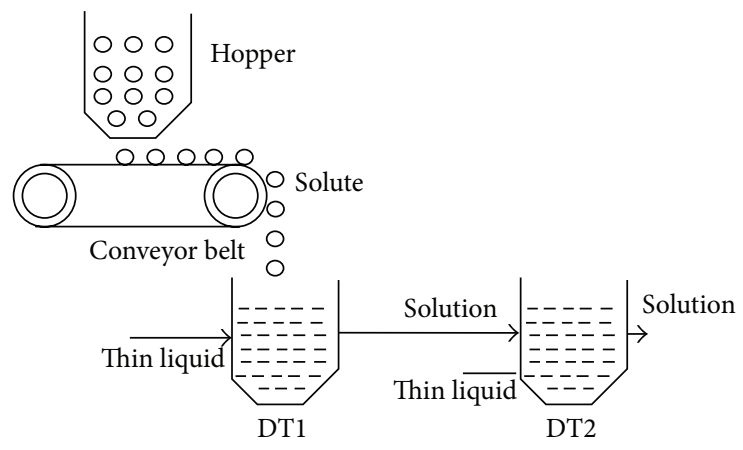

Figure 1: Bipolar dissolving tank (DT) in chemical process.

$x_{1}(t)=\rho_{1}(t)-\rho_{10}, x_{2}(t)=\rho_{2}(t)-\rho_{20}$. Above all, the concentration of the dissolving tank satisfies the following system:

$$
\begin{aligned}
{\left[\begin{array}{l}
X_{1} \dot{x}_{1}(t) \\
X_{2} \dot{x}_{2}(t)
\end{array}\right]=} & {\left[\begin{array}{cc}
-\frac{a+\delta a}{V} & 0 \\
0 & -\frac{a+\delta a}{V}
\end{array}\right]\left[\begin{array}{l}
x_{1}(t) \\
x_{2}(t)
\end{array}\right] } \\
& +\left[\begin{array}{cc}
0 & 0 \\
\frac{a+\delta a}{p V} & 0
\end{array}\right]\left[\begin{array}{l}
x_{1}(t-\tau) \\
x_{2}(t-\tau)
\end{array}\right] \\
& {\left[\begin{array}{cc}
0 & 0 \\
\frac{1-p}{p} & 0
\end{array}\right]\left[\begin{array}{l}
\dot{x}_{1}(t-\tau) \\
\dot{x}_{2}(t-\tau)
\end{array}\right]+\left[\begin{array}{l}
1 \\
1
\end{array}\right] \omega } \\
+ & {\left[\begin{array}{cc}
1 & 0 \\
0 & \frac{p}{V}
\end{array}\right]\left[\begin{array}{l}
u_{1}(t) \\
u_{2}(t)
\end{array}\right], } \\
y(t) & =\left[\begin{array}{ll}
0 & 1
\end{array}\right]\left[\begin{array}{l}
x_{1}(t) \\
x_{2}(t)
\end{array}\right]+\omega .
\end{aligned}
$$

Let $a$ be $1500 \mathrm{~L} / \mathrm{h} \approx 0.4167 \mathrm{~L} / \mathrm{s}, p \approx 97.0 \%, V=200 \mathrm{~L}, \delta a=$ $0.5 \sin t$

Obviously, the uncontrolled system is not a stable system. For the system with disturbance, if the system is passive, then it is internal stability. It is easy to know that the above uncontrolled system does not satisfy the condition of theory 2. That is to say, this system is not output strictly passive in terms of theory 2 . Then the output strictly passive controller is designed such that the resulting closed-loop system satisfied the prescribed passive performance so that the closed-loop system is to maintain internal stability.

Type 1: the perturbations in additive form (17) and (18) are

$$
\begin{gathered}
M_{a}=0.1, \quad L_{a}=\left[\begin{array}{l}
0.1 \\
0.2
\end{array}\right]^{T}, \\
L_{a 1}=\left[\begin{array}{l}
0.1 \\
0.4
\end{array}\right]^{T}, \quad F_{a}(t)=\cos t .
\end{gathered}
$$

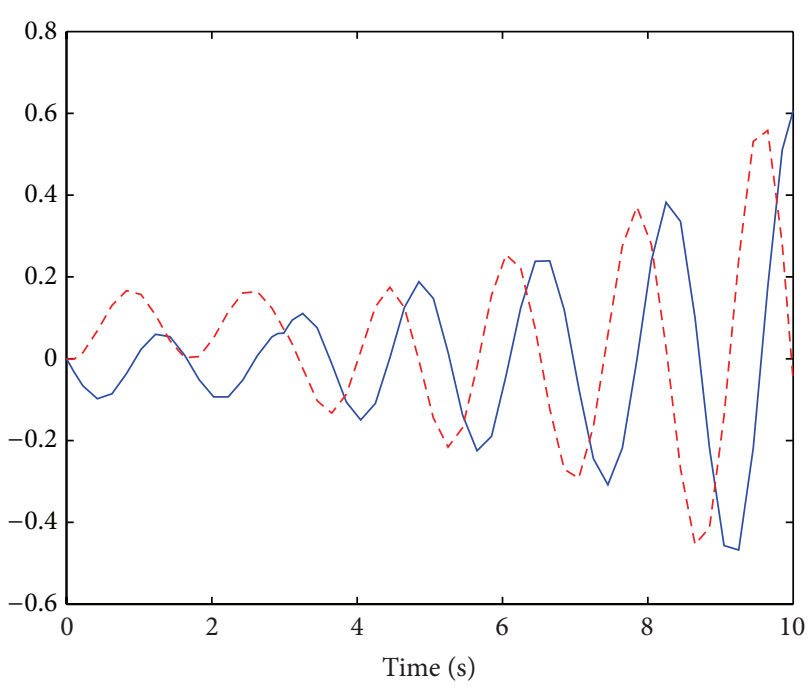

FIGURE 2: State response of uncontrolled system $x_{1}$ (solid line) and $x_{2}$ (dashed line).

Suppose that $\delta_{0}=0.2$; we could get the solutions to (40)-(43), which are as follows:

$$
\begin{gathered}
P=\left[\begin{array}{ll}
2.4663 & 0.8714 \\
0.8714 & 1.1222
\end{array}\right], \quad T_{1}=\left[\begin{array}{ll}
-7.8872 & 5.1266
\end{array}\right], \\
T_{2}=\left[\begin{array}{ll}
-2.6041 & 2.7617
\end{array}\right] .
\end{gathered}
$$

This solution leads to the following output strictly passive controller gains:

$$
K_{a}=\left[\begin{array}{ll}
-6.6314 & 9.7177
\end{array}\right], \quad K_{a 1}=\left[\begin{array}{ll}
-2.6533 & 4.5213
\end{array}\right] .
$$

Type 2: the perturbations in multiplicative form (19) and (20) are

$$
H_{m}=-1, \quad L_{m}=0.5, \quad F_{m}(t)=\cos t .
$$

Solving the LMI (44) to (45) still with $\delta_{0}=0.2$ we have

$$
\begin{gathered}
P=\left[\begin{array}{ll}
2.2783 & 1.4506 \\
1.4506 & 1.7164
\end{array}\right], \quad T_{1}=\left[\begin{array}{ll}
-47.9123 & 3.6942
\end{array}\right], \\
T_{2}=\left[\begin{array}{ll}
-13.6186 & 1.6788
\end{array}\right] .
\end{gathered}
$$

This solution leads to the following output strictly passive controller gains:

$$
\begin{aligned}
& K_{a}=\left[\begin{array}{ll}
-6.6314 & 9.7177
\end{array}\right], \\
& K_{a 1}=\left[\begin{array}{ll}
-2.6533 & 4.5213
\end{array}\right] .
\end{aligned}
$$

Figure 2 is the state response of uncontrolled system (46) when the initial condition $x(0)=\left[\begin{array}{ll}0 & 0\end{array}\right]^{T}$ and the disturbance input $\omega(t)=\sin t$. Figure 3 is the state response of the closed-loop system under the designed output strictly passive controller (16) with additive form when the initial condition 


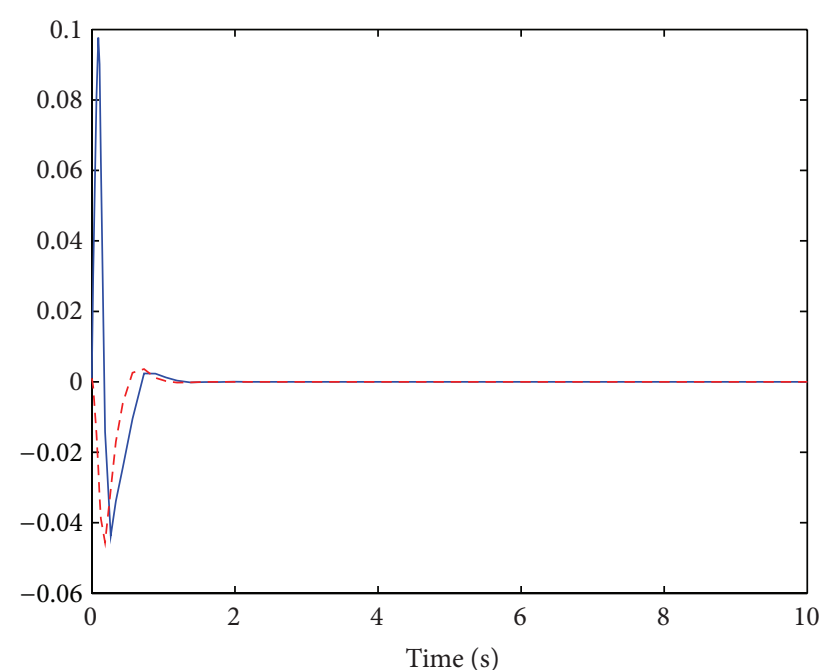

FIGURE 3: State response of the closed-loop system with additive form $x_{1}$ (solid line) and $x_{2}$ (dashed line).

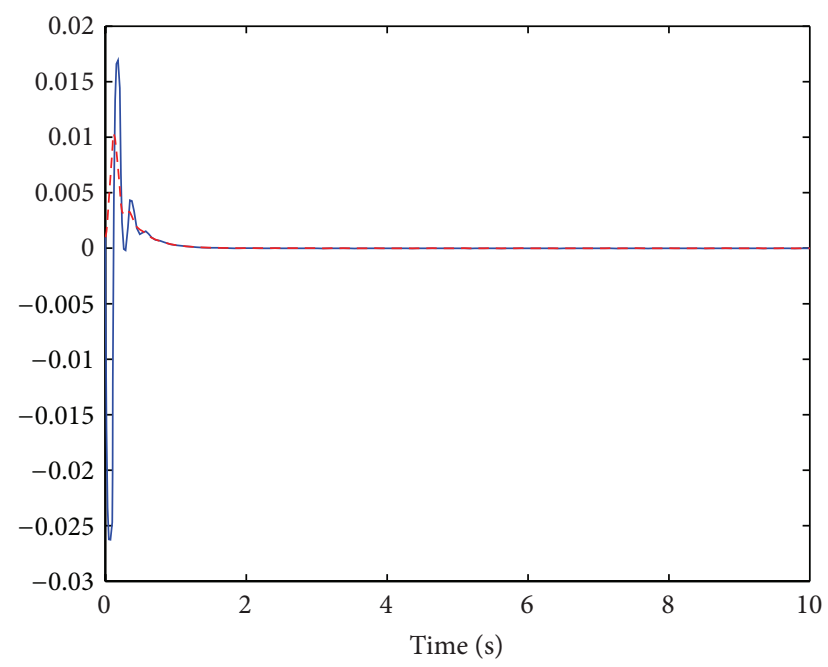

FIGURE 4: State response of the closed-loop system with multiplicative form $x_{1}$ (solid line) and $x_{2}$ (dashed line).

$x(0)=\left[\begin{array}{ll}0.001 & 0.001\end{array}\right]^{T}$ and the disturbance input $\omega(t)=$ $\sin t$. Figure 4 is the state response of the closed-loop system under the designed output strictly passive controller (16) with multiplicative form when the initial condition $x(0)=$ $\left[\begin{array}{ll}0.001 & 0.001\end{array}\right]^{T}$ and the disturbance input $\omega(t)=\sin t$.

\section{Conclusions}

This paper has established a new version of bounded real lemma for neutral singular systems of LMIs. In this conclusion strict LMI is obtained. This overcomes the shortcoming which may result in numerical problems which are fragile and usually not satisfied perfectly. Design of the state feedback output strictly passive controller such that the closed-loop system is output strictly passivity. Finally, a numerical example illustrates the ability of this kind of passive controller.

\section{Conflict of Interests}

The authors declare that there is no conflict of interests regarding the publication of this paper.

\section{Acknowledgments}

This research is supported by National Natural Science Foundation of China (Grant no. 61203214), Provincial Science and Technology Department of Education projects, the general project (L2013101).

\section{References}

[1] Y.-P. Huang and K. Zhou, "Robust stability of uncertain timedelay systems," IEEE Transactions on Automatic Control, vol. 45, no. 11, pp. 2169-2173, 2000.

[2] M. S. Mahmoud, Robust Control and Filtering for Time-Delay Systems, vol. 5 of Control Engineering Series, Marcel Dekker, New York, NY, USA, 2000.

[3] S.-C. Tong, X.-L. He, and H.-G. Zhang, "A combined backstepping and small-gain approach to robust adaptive fuzzy output feedback control," IEEE Transactions on Fuzzy Systems, vol. 17, no. 5, pp. 1059-1069, 2009.

[4] Y.-J. Liu, S. Tong, and C. L. P. Chen, "Adaptive fuzzy control via observer design for uncertain nonlinear systems with unmodeled dynamics," IEEE Transactions on Fuzzy Systems, vol. 21, no. 2, pp. 275-288, 2013.

[5] Y. Chen, W. X. Zheng, and A. Xue, "A new result on stability analysis for stochastic neutral systems," Automatica, vol. 46, no. 12, pp. 2100-2104, 2010.

[6] S.-I. Niculescu and B. Brogliato, "Force measurement timedelays and contact instability phenomenon," European Journal of Control, vol. 5, no. 2-4, pp. 279-289, 1999.

[7] K. Engelborghs, M. Dambrine, and D. Roose, "Limitations of a class of stabilization methods for delay systems," IEEE Transactions on Automatic Control, vol. 46, no. 2, pp. 336-339, 2001.

[8] X. Li, W. Xing, and Q. Zhang, "Robust strictly dissipative control for uncertain neutral stochastic time-delay systems," in Proceedings of the 3rd International Conference on Intelligent Control and Information Processing (ICICIP '12), pp. 454-457, Dalian, China, July 2012.

[9] Y. He, M. Wu, J.-H. She, and G.-P. Liu, "Delay-dependent robust stability criteria for uncertain neutral systems with mixed delays," Systems and Control Letters, vol. 51, no. 1, pp. 57-65, 2004.

[10] J.-J. Yan, M.-L. Hung, and T.-L. Liao, "An EP algorithm for stability analysis of interval neutral delay-differential systems," Expert Systems with Applications, vol. 34, no. 2, pp. 920-924, 2008.

[11] J. Kuang, H. Tian, and K. Shan, "Asymptotic stability of neutral differential systems with many delays," Applied Mathematics and Computation, vol. 217, no. 24, pp. 10087-10094, 2011.

[12] M. S. Mahmoud, "Robust $H_{\infty}$ control of linear neutral systems," Automatica, vol. 36, no. 5, pp. 757-764, 2000.

[13] S. Xu, J. Lam, Y. Zhou, and M. Sun, "Control for neutral systems via Non-fragile state feedback controller," in International Conference on Control, Automation, Robotics and Vision, pp. 48-51, Kunming, China, December 2004. 
[14] A. Shariatia, H. D. Taghirad, and A. Fatehi, "A neutral system approach to $H_{\infty} \mathrm{PD} / \mathrm{PI}$ controller design of processes with uncertain input delay," Mathematical Problems in Engineering, vol. 24, no. 3, pp. 144-157, 2014.

[15] H. Li, Robust Control for Neutral Systems, Northwestern Polytechnical University Press, 2006.

[16] H. Shen, S. Xu, X. Song, and G. Shi, "Passivity-based control for Markovian jump systems via retarded output feedback," Circuits, Systems, and Signal Processing, vol. 31, no. 1, pp. 189-202, 2012.

[17] L. Cai-na and C. Bao-tong, "Robust passive control of uncertain linear neutral systems with time-delays," Mathematica Applicata, vol. 20, no. 2, pp. 361-369, 2007.

[18] H. Hu, T. Zhang, and H. Zhang, "Passive control for uncertain neutral systems via state feedback with memory," in Proceedings of the 31st Chinese Control Conference (CCC '12), pp. 1008-1012, Hefei, China, July 2012.

[19] H. Hu, D. Zhao, and Q. Zhang, "Observer-based decentralized control for uncertain interconnected systems of neutral type," Mathematical Problems in Engineering, vol. 2013, Article ID 134935, 12 pages, 2013.

[20] J. Yang, S. Zhong, and L. Xiong, "A descriptor system approach to non-fragile $H_{\infty}$ control for uncertain fuzzy neutral systems," Fuzzy Sets and Systems, vol. 160, no. 4, pp. 423-438, 2009.

[21] J. Ren and Q. Zhang, "Non-fragile PD state $H_{\infty}$ control for a class of uncertain descriptor systems," Applied Mathematics and Computation, vol. 218, no. 17, pp. 8806-8815, 2012.

[22] Q.-X. Lan, C.-Y. Feng, and J.-R. Liang, "State feedback stabilization of neutral type descriptor systems," in Proceedings of the WRI World Congress on Computer Science and Information Engineering (CSIE '09), pp. 247-251, April 2009.

[23] H. Li, H.-B. Li, and S.-M. Zhong, "Stability of neutral type descriptor system with mixed delays," Chaos, Solitons \& Fractals, vol. 33, no. 5, pp. 1796-1800, 2007.

[24] H. Wang and A. Xue, "Delay-dependent robust stability criteria for neutral singular systems with time-varying delays and nonlinear perturbations," in Proceedings of the American Control Conference (ACC '09), pp. 5446-5451, St. Louis, Mo, USA, June 2009.

[25] Y.-J. Liu, S.-C. Tong, and T.-S. Li, "Observer-based adaptive fuzzy tracking control for a class of uncertain nonlinear MIMO systems," Fuzzy Sets and Systems, vol. 164, no. 1, pp. 25-44, 2011.

[26] S. C. Tong, Y. Li, Y. M. Li, and Y. J. Liu, "Observer-based adaptive fuzzy backstepping control for a class of stochastic nonlinear strict-feedback systems," IEEE Transations on Systems, Man and Cybernetics, Part B: Cybernetics, vol. 41, no. 6, pp. 1693-1704, 2011.

[27] Y. Zhao and Y. Ma, "Stability of neutral-type descriptor systems with multiple time-varying delays," Advances in Difference Equations, vol. 2012, article 15, 2012.

[28] J. K. Hale, Theory of Functional Differential Equations, Springer, New York, NY, USA, 1977.

[29] W. Jiang, Degenerate Differential System with Delay, Anhui University Press, Anhui, China, 1998.

[30] C. E. de Souza and X. Li, "Delay-dependent robust $H_{\infty}$ control of uncertain linear state-delayed systems," Automatica, vol. 35, no. 7, pp. 1313-1321, 1999.

[31] J. Wang, Passivity-Based Control Theory and Its Applications, Publishing House of Electronics Industry, Beijing, China, 2010.

[32] S. Xu, Robust Control and Filtering of Singular Systems, Springer, New York, NY, USA, 2006.
[33] Q. Li, Q. Zhang, and J. Wang, "Non-fragile observer-based passive control for descriptor systems with time-delay," Journal of Control Theory and Applications, vol. 7, no. 3, pp. 237-242, 2009. 


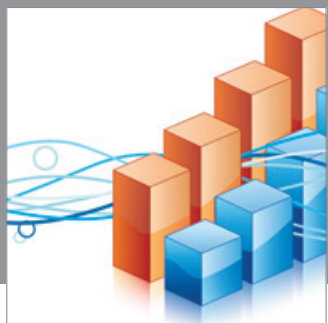

Advances in

Operations Research

mansans

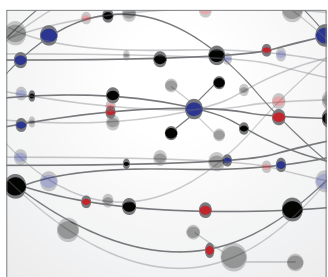

The Scientific World Journal
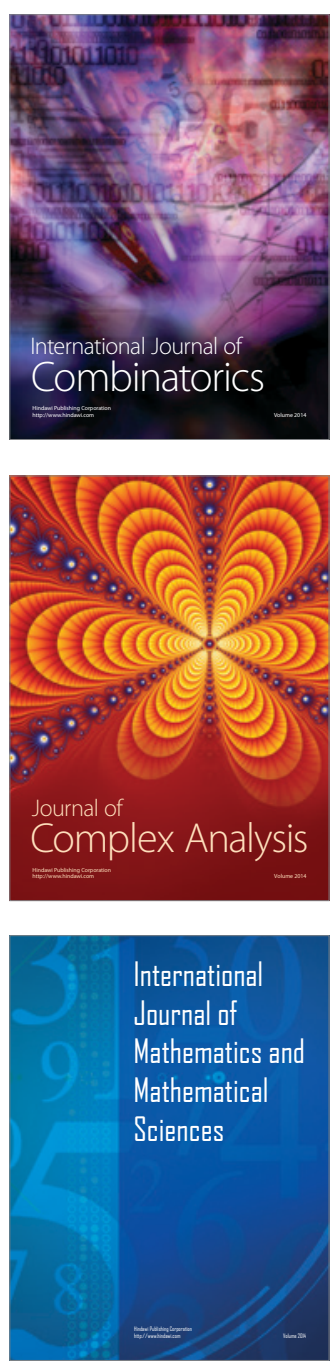
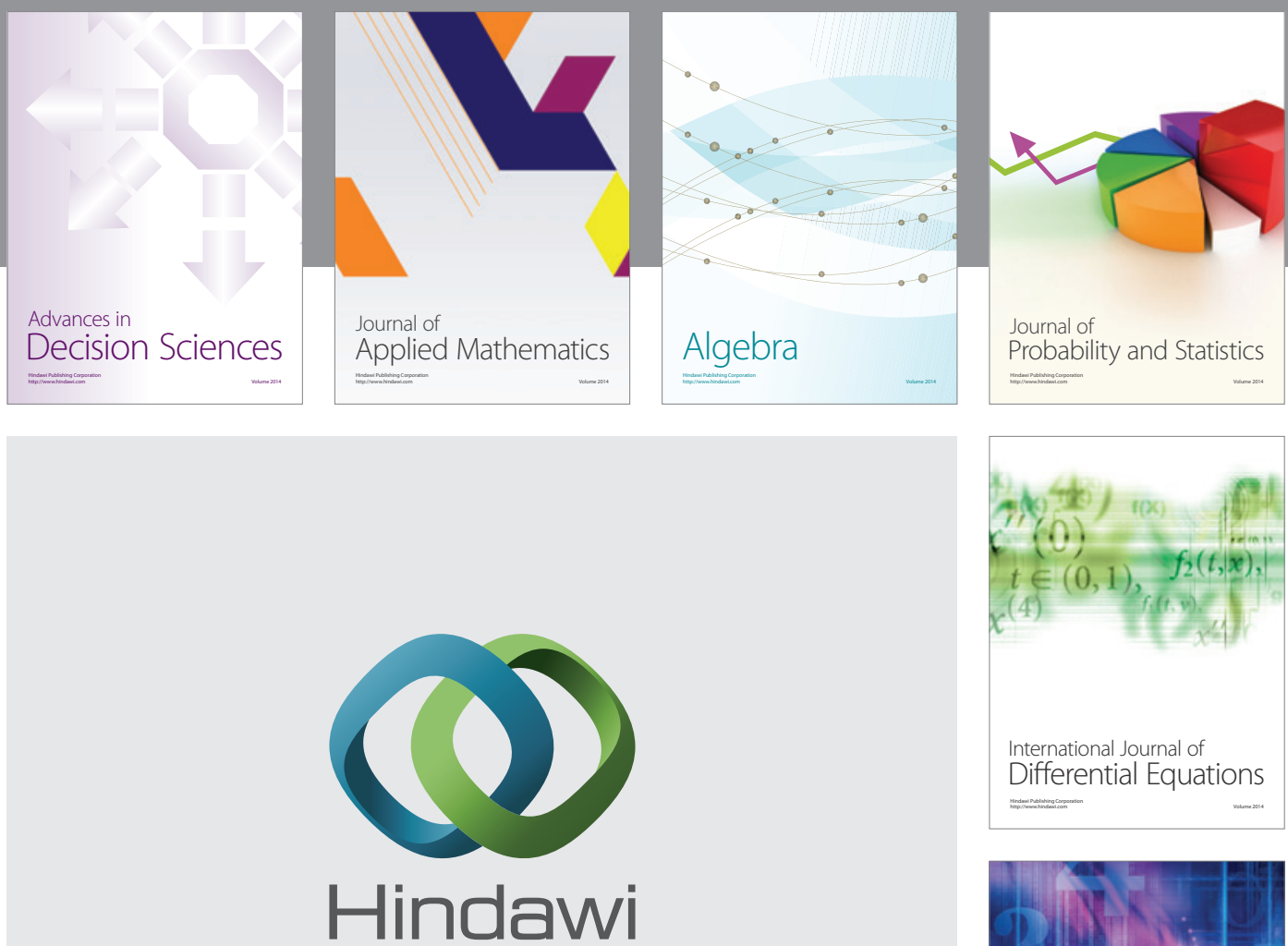

Submit your manuscripts at http://www.hindawi.com
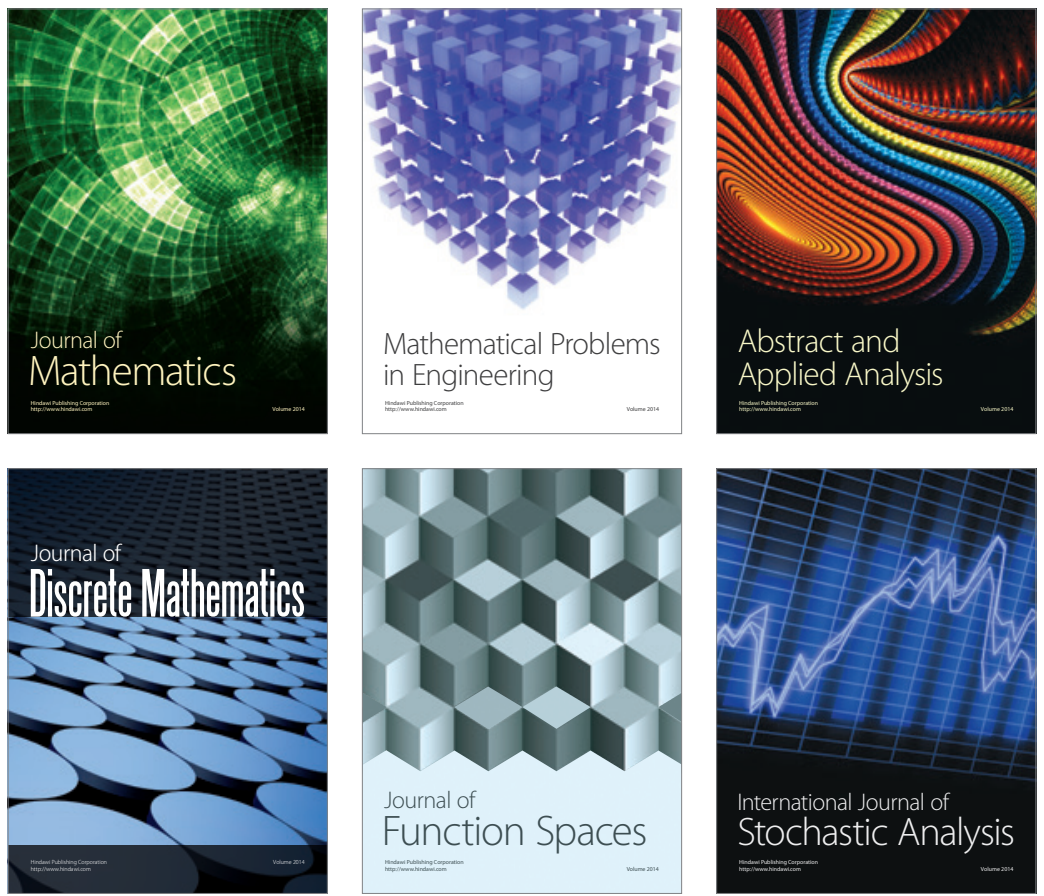

Journal of

Function Spaces

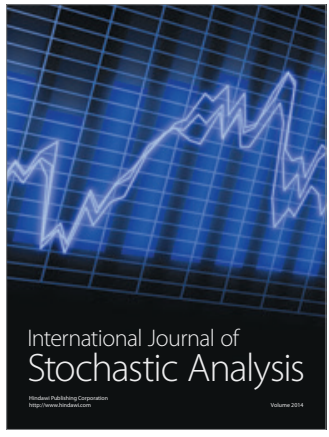

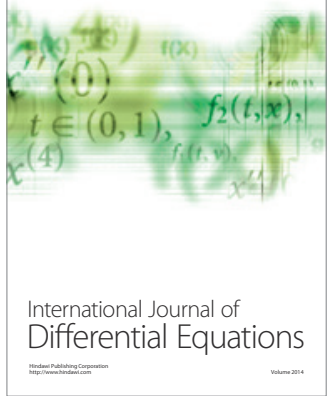
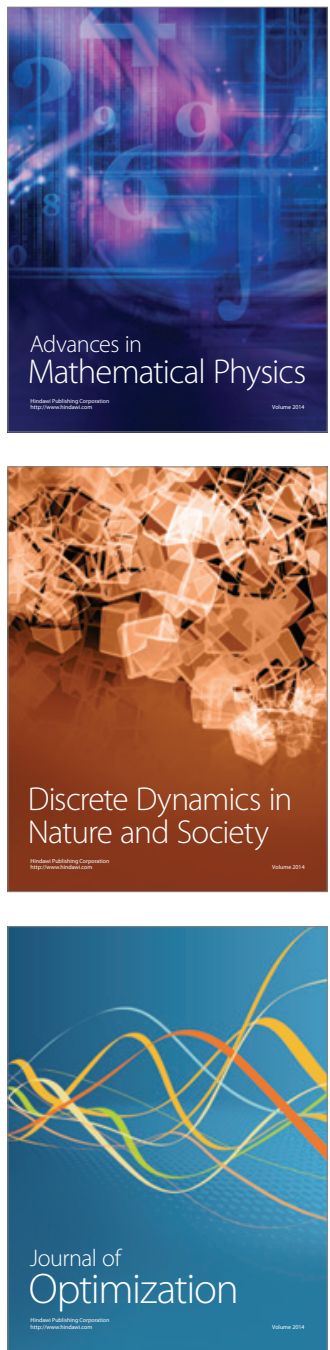